NBER WORKING PAPER SERIES

\title{
FEEDBACK AND THE USE OF CURRENT INFORMATION: \\ THE USE OF GENERAL LINEAR POLICY RULES \\ IN RATIONAL EXPECTATIONS MODELS
}

\author{
Willem H. Buiter
}

Working Paper No. 335

\author{
NATIONAL BUREAU OF ECONOMIC RESEARCH \\ 1050 Massachusetts Avenue \\ Cambridge, MA 02138
}

April 1979

The research reported here is part of the NBER's research program in International Studies. Any opinions expressed are those of the author and not those of the National Bureau of Economic Research. 
Feedback and the Use of Current Information:

The Use of General Linear Policy Rules in Rational Expeciations Modols

\begin{abstract}
The behavior of several stochastic dynamic rational expectations models is studied when policy behavior can be described by a linear rule. Four policy components are distinguished: a current response component, a feedback component, an open-1oop component and a stochastic component. Policy is evaluated in terms of the current and asymptotic first and second moments of the state variables. The importance of distinguishing between variability and uncertainty is brought out. The conditional variance is argued to be the appropriate measure of uncertainty. The analysis is applied to a model of foreign exchange market intervention.
\end{abstract}

\author{
Willem H. Buiter \\ Woodrow Wilson School of Public \\ and International Affairs \\ Princeton University \\ Princeton, N.J. 08540 \\ (609) $452-4816$
}




\section{Introduction}

In a number of recent papers (Kareken, Muench and Wallace [1973], LeRoy [1975], Friedman [1975, 1977], Wog] om [1979], Black [1976, 1977], Boyer [1978] Roper and. Turnovsky [1978], Siegel [1978] and Woglom [1979]), the ability of the policy authorities to stabilize the economy has been shown to depend in an important way on the extent to which the current value of the policy instrument(s) can be made a function of current random disturbances--more precisely of the innovations in these disturbances. This issue is especially important for financial policy and foreign exchange market intervention and exchange rate management. Financial policy instruments, be they financial asset stocks, interest rates or exchange rates can be adjusted virtually continuously and costlessly. Some financial market data (e.g., exchange rate observations, stock prices and transactions in government debt) are observable and available on a fairly current basis. Such currently observable data provide information about the sources of random shocks to the economy. Financial policy should be conducted in such a way as to extract the information contained in observable financial market data and exploit it in achieving the authorities' stabilization objectives.

In this paper I evaluate the effect of general linear policy rules on the behavior of a number of stochastic linear rational expectations models. This extends the analysis of Aoki and Canzoneri [1979] who only consider feedback policy. The implications of the policy rules are evaluated by considering the first two moments of the joint distribution of the state variables of the model. The short-run policy effects are measured by the single period mean and variance. The long-run policy effects are evaluated by considering the steady-state mean and variance (see also Turnovsky [1976]). 
The importance of distinguishing between variability and uncertainty is brought out. The current period variance can be viewed as the sim of an anticipated component and an unanticipated component. By making use of the information contained in currently observed variables the policy authority can reduce--and in the simple models of Poole, Boyer and Siegel eliminate-the anticipated variance of the target variables. It cannot affect the unanticipated component. This unanticipated component is, as Siegel [1978] points out, given by the conditional variance--the conditional second moment about the conditional mean, where the conditioning information set is the subset of the state that is currently observed.

The formal analysis is presented in Section 2. An application to Boyer's model of foreign exchange market intervention complements the theoretica1 exposition.

\section{Genera1 Linear Policy Rules in Linear Rationa1 Expectations Models}

The simplest possible linear rational expectations model is given in equation (1).

$$
z_{t}=A z_{t-1}+B_{1} z_{t-1}+C x_{t}+u_{t}
$$

$z_{t}$ is a vector of endogenous (or state) variables. $x_{t}$ is a vector of policy instruments. $u_{t}$ is a vector of i.i.d. random disturbances with a zero mean vector and contemporaneous variance-covariance matrix $\Sigma_{u}$. $z_{t \mid t-1}$ is the rational expectation of $t$ as of $t-1$. It is defined by $\left.z_{t}\right|_{t-i}=E\left(\left.z_{t}\right|_{t-i}\right)$. $I_{t-i}$ is the information set, common to the public and private sectors, available at the beginning of period $t-i$. $E$ is the mathematical expectation operator. Equation (1) will be generalized in three directions represented in equations (1'), (1") and (1'').

(1' $\quad z_{t}=A z_{t-1}+B_{1} z_{t-1}+\left.B_{2} z_{t}\right|_{t-2}+C x_{t}+u_{t}$. 
In equation ( $\left.1^{\prime}\right)$, if policy at $t$ can be based on information that became available in $t-1$ (or $t$ ), it will he based on more recent information than was available when private agents formed expectations of $z_{t}$ in period $t-2$, even if at each point in time, public and private agents have the same information available. Multi-period nominal wage or price contracts can generate models like ( $\left.{ }^{\prime}\right)$.

$$
z_{t}=A z_{t-1}+B_{1} z_{t \mid t-1}+B z_{t+1 \mid t}+C x_{t}+u_{t}
$$

In (1") the current value of $z_{t}$ depends on current expectations of the future and on past expectations of the present. An example of such a model is an IS-LM model with a Lucas supply function. If $\mathrm{p}_{t}$ denotes the $\log$ of the price level, current real output is a function of $P_{t}-P_{t} \mid t-1$. The real interest rate will be a function of $p_{t+1 \mid t}-p_{t}$. In (1'") agents last period made forecasts two periods into the future.

(1'") $\quad z_{t}=A z_{t-1}+B z_{t+1 \mid t-1}+C x_{t}+u_{t}$

Policy behavior is specified in equation (2)

$$
x_{t}=G_{t}^{0} z_{t}+G_{t}^{1} z_{t-1}+\bar{x}_{t}+v_{t} \cdot \underline{1 /}
$$

$G_{t}^{0}{ }^{2}$ is the "current response component" of the policy rule. Current realizations of the state partly determine the current value of the instrument. This will be interpreted in more detail below. $G_{t}^{1} z_{t-1}$ is the "feedback component" of the policy rule $\frac{2 /}{} \bar{x}_{t}$ is the non-stochastic "open loop component" of the policy rule. It is known in advance for all future time. $v_{t}$ is a random vector representing the "stochastic component" of the policy rule. ${ }^{3 /}$ It is i.i.d. with a zero mean vector. Its contemporaneous variance-covariance matrix is $\Sigma_{\mathrm{v}}$ and its contemporaneous covariance matrix 
with $u_{t}$ is given by $\ddot{z}_{u v}$. The information set available to both sectors is given in (3)

$$
I_{t-i} \equiv\left\{G_{t}^{0}, G_{t}^{1}, z_{t-i}, x_{t-i}, z_{t-i}, x_{t-i-u}, \bar{x}_{t}, \Sigma_{u}, \Sigma_{v}, \Gamma_{u v}\right\} . i \geq 0
$$

Thus both sectors know the deterministic and stochastic structure of the model. Policy behavior can be forecast using (4).

$$
x_{t \mid t-i}=G_{t}^{0} z_{t-i}+G_{t}^{1} z_{t-1 \mid t-i}+\bar{x}_{t} \cdot \quad i>0
$$

The model of equation 1 contains the "policy surprise model," according to which only unanticipated policy matters, as a special case. Note that $e_{t}$ can be contained in $z_{t}$. If the submatrix $b_{1}$ of $B_{1}$ corresponding to $\left.x_{t}\right|_{t-1}$ is equal to the negative of the $C$ matrix, policy enters the model on $1 y$ as $C\left(x_{t \mid t-1}-x_{t}\right)$.

The rational expectations solution of the model given in (1), (2) and (3) is given in (5).

(6c) $\quad e_{t}=\left(I-C G_{t}^{0}\right)^{-1}\left(C_{t}+u_{t}\right) \quad \underline{4}$

Aoki and Canzoneri analyze the special case of this model when the policy rule is simplified by setting $G_{t}^{0}$ and $\bar{x}_{t}$ identically equal to zero. Considerable interest attaches to the dependence of the first two moments of this stochastic difference equation on the various components of the policy rule. It is to this that we now turn. 
By successive substitution in (5), $z_{t}$ can be expressed as in (7):

(7) $\quad z_{t}=\prod_{i=0}^{t-1} \tilde{A}_{t-i} z_{0}+e_{t}+\sum_{j=0}^{t-2} \prod_{i=0}^{j} \tilde{A}_{t-i}{ }^{c} t-j-1+\tilde{C}_{t} \bar{x}_{t}+\sum_{j=0}^{t-2} \prod_{i=0}^{j} \tilde{A}_{t-i} \tilde{C}_{t-j-1} \bar{x}_{t-j-1}$

If the policy matrices $G_{t}^{0}$ and $G_{t}^{1}$ were time-invariant, i.e., $G_{t}^{0}=G^{0}$ and

$G_{t}^{1}=G^{1}$, and if the open-loop component $\bar{x}_{t}$ were a constant $\bar{x}$, equation (7)

simplifies to:

(7') $z_{t}=\tilde{A}^{t} z_{0}+\sum_{j=0}^{t-1} \tilde{A}^{j} e_{t-j}+\sum_{j=0}^{t-1} \tilde{A} \tilde{j} \vec{C}$

The expectation $E\left(z_{t}\right)$ is given by

(8) $\quad E\left(z_{t}\right)=\bar{z}_{t}=\tilde{A}^{t} z_{0}+\sum_{j=0}^{t-1} \tilde{A} \tilde{j} \bar{x}$

If all characteristic roots of $\tilde{A}$ have modulus less than unity, the mean function $\bar{z}_{t}$ will, as $t$ approaches infinity, approach the limit:

(9) $\lim _{t \rightarrow \infty} \bar{z}_{t}=\bar{z}=[I-\tilde{A}]^{-1} C \bar{x}$

Thus the asymptotic or steady state mean function will in general depend on three of the four policy components: the current response component $\left(G^{0}\right.$ in $\left.\tilde{A}\right)$, the feedback component $\left(G^{1}\right.$ in $\left.\tilde{A}\right)$ and the open-loop component. The stochastic disturbance term $e_{t}$ in (5) is i.i.d. with a zero mean vector and contemporaneous variance-covariance matrix $\Sigma_{e}$ given by

$$
\Sigma_{e}=\left(I-C G_{t}^{0}\right)^{-1}\left[C \Sigma_{v} C^{\prime}+C \Sigma_{u v}+\Sigma_{u v} C^{\prime}+\Sigma_{u}\right]\left(I-C G_{t}^{0}\right)^{-1}
$$

Let $z_{t}^{*}$ denote the deviation of the state variable $z_{t}$ from its unconditional mean, i.e. $z_{t}^{*}=z_{t}-\bar{z}_{t}$. The unconditional variance of $z_{t}$, for the case in which $G_{t}^{0}$ is time-invariant is given in (11). 


$$
E\left(z_{t}^{*} z_{t}^{*^{\prime}}\right)=\Sigma_{e}+\tilde{A} \Sigma_{e} \tilde{A}^{\prime}+\tilde{A}^{2} \Sigma_{e} \tilde{\Lambda}^{\prime 2}+\ldots \cdot \cdot+\tilde{\Lambda}^{t-1} \Sigma_{e^{\prime}} \tilde{A}^{t-1}
$$

The unconditional asymptotic variance of $z_{t}$ is given by

$$
\lim _{t \rightarrow \infty} E\left(z \underset{t}{*} z_{t}^{\dot{*}^{\prime}}\right)=\Sigma_{z}=\Sigma_{e}+\tilde{A} \Sigma_{e^{A}} \tilde{A}^{\prime} \tilde{A}^{2} \Sigma_{e} \tilde{\Lambda}^{\prime^{2}}+\cdots \cdot
$$

If the eigenvalues of $\tilde{A}$ all have modulus less than unity, the right-hand side of (12) will converge to the expression given in (13).

$$
\Sigma_{z}=\Phi \Omega \Phi^{\prime}
$$

$\Omega$ is a matrix whose typical component $w_{i j}$ is given by $w_{i j}=\mu_{i j}\left(1-\lambda_{i} \lambda_{j}\right)^{-1}$. $\lambda_{i}$ is the $i^{\text {th }}$ characteristic root of $\tilde{A} . \mu_{i j}$ is the typical component of the matrix $M$ which is defined by: $M=\Phi^{-1} \Sigma_{e \Phi^{\prime-1}}$ or $\Phi M \Phi^{\prime}=\Sigma_{e^{*}}$ denotes a matrix consisting of columns of the right-characteristic vectors of $\tilde{A}$. Note that $\Sigma_{e}$ depends on the current response matrix $G^{0}$ and on the second moment of the stochastic component of policy, $\Sigma_{v}$ and $\Sigma_{u v}$. Even if the current response matrix were the zero matrix and even if policy behavior were non-stochastic $\left(\Sigma_{\mathrm{v}}=\Sigma_{\mathrm{uv}}=0\right)$, policy will affect the asymptotic variance of $z$, because $\Sigma_{z}$ depends not only on $\Sigma_{e}$, but also on $\tilde{A}$, which is a function of the feedback component $G^{3}$. Both the first and second moments of the steady-state distribution of $z_{t}$ will therefore in general depend on the deterministic components of the policy rule, even if there is no current response of the instrument vector to the state vector.

The unconditional single-period mean and variance of $z_{t}$

From equation (5) it is easily seen that the unconditional single-period (or current-period) expectation of $z_{t}$ is:

$$
E\left(z_{t}\right)=\tilde{A}_{t} z_{t-1}+\tilde{C}_{t} \bar{x}_{t}
$$


This is a function of the current response component, $c_{t}^{0}$ in $\tilde{A}_{t}$ and $\tilde{C}_{t}$, of the feedback component $G_{t}^{1}$ in $\tilde{\Lambda}_{t}$ and of the open-joop component $\ddot{x}_{t}$.

The unconditional current-period or single-period variance of $z_{t}$ is

$$
E\left(z_{t}^{*} z_{t}^{* \prime}\right)=E\left(e_{t} e_{t}^{\prime}\right)=\Sigma_{e, t}
$$

Thus the single-period unconditional variance of ${ }^{2} t$ depends on the current response component, $G_{t}^{0}$, and on the stochastic component, $\Sigma_{u}$ and $\Sigma_{u v}$. I.t is independent of the open-loop component $\bar{x}_{t}$ and of the feedback component $G_{t}^{1}$. Boyer [1.978], Roper and Turnovsky [1978] and Black [1976, 1977] analyze foreign exchange market policies aimed at minimizing the unconditional single-period variance of real income. Woglom [1979] analyzes a rational expectations extension of Poole's model (Poole [1.970]) in which a monetary policy rule is chosen that minimizes the single-period unconditional variance of real income. The policy rule consists of a current response component and a feedback component. Woglom's conclusion that the variance of real income is independent of the feedback component is correct for the single-period variance but not for the asymptotic variance.

In general, the stabilization policies pursued by the authorities can be represented as the selection of values for the policy matrices that will. minimize some bilinear form of the appropriate variance-covariance matrix, i.e. minimize $w_{1} \Sigma_{z} w_{2}$ with respect to $\hat{G}^{0}$ and $G^{l}$ or minimize $w_{1} \Sigma_{2} w_{2}$ with respect to $\hat{G}^{0}$. $w_{1}$ is a row vector, $w_{2}$ a column vector.

The conditional single-period mean and variance of $\mathrm{z}_{\mathrm{t}}$

The interpretation of the current response component of policy is by no means obvious. The value of the policy instruments, in period $t, x_{t}$, is made contingent on the realized value of the state vector in period $t, z_{t}$. This realized or equilibrium value of the state vector is in turn co-determined 
by the value assigned to the policy instrument vector in $t$. The ability to plan and execute current policy decisions contingent on the current value of the state vector, requires that the state vector can be currently observed, wholly or partly. Current policy is determined on the basis of at least partial information about the current state. Such partial information about the current state can be modelled in many ways. One natural way to proceed is to take an errors-in-variables approach: the policy maker at time $t$ does not observe $z_{t}$ but $\hat{z}_{t}=z_{t}+\varepsilon_{t}$, where $\varepsilon_{t}$ is a random, i.i.d. observation error. If the true policy rule were $x_{t}=G_{t}^{0^{\wedge}}{ }_{t}+G_{t}^{1} z_{t-1}+\bar{x}_{t}$, this would provide an explanation of the error term $v_{t}$ in the policy function, because the policy rule can now be rewritten as: $x_{t}=G_{t}^{0}{ }_{t}+G_{t}^{1} z_{t-1}+$ $\bar{x}_{t}+v_{t}$, where $v_{t}=G_{t}^{0} \varepsilon_{t}$. This is not the approach adopted in this paper. Instead I shall assume that when the response of $x_{t}$ to $z_{t}$ is decided, the policy authority observes some linear combination of the current state, i.e. he observes $\Psi_{t}{ }^{z} t$. If $\Psi_{t}$ is invertible, knowledge of $\Psi_{t}{ }_{t}$ is equivalent to the observation of $z_{t}$, as the policy authority knows $\Psi_{t}$. We do not assume $\Psi_{t}$ to be invertible but we do assume it to be of full rank. The current response of the policy instrument is a linear function $\bar{G}_{t}^{0}$ of this currently accrued information. Thus the current response matrix $G_{t}^{0}$ can be viewed as the product of the current information matrix $\Psi_{t}$ and the true current policy response matrix $\bar{G}_{t}^{0}$.

$$
G_{t}^{0}=\bar{G}_{t}^{0} \Psi_{t}
$$

If the private sector has access to the same partial information as the public sector, and if it is uncertainty about ${ }_{t}$ rather than merely variability of $z_{t}$ that matters, the conditional variance of $z_{t}$ rather than its unconditional variance should be evaluated, with 'conditional' referring to the knowledge of $\Psi_{t}{ }^{z}$. 
The conditional first moment $E\left({ }{ }_{t} \mid y_{t} z_{t}\right)$ is found quite easily.

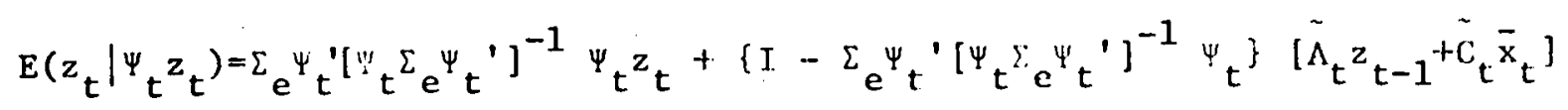

Thus the conditional expectation of $z_{t}$ is a weighted average of the unconditional expectation and the current partial observation on $z_{t}$. Consider some special cases. In the information matrix $\psi_{t}$ is invertible, $E\left(z_{t} \mid \psi_{t} z_{t}\right)=z_{t}$, as if the complete state is currently observed. If the information matrix is the null matrix, $E\left(z_{t} \mid \Psi_{t} z_{t}\right)=\tilde{A}_{t} z_{t-1}+\tilde{C}_{t} x_{t}$. When there is no information conditioning the forecast, the conditional expectation equals the unconditional expectation. Note that unless there is full current information, the conditional expectation will depend on $G_{t}^{0}, G_{t}^{1}, \bar{x}_{t}, \Sigma_{v}$ and $\Sigma_{u v}$.

The conditional variance of $z_{t}$ that we are interested in is given in (18). $I_{t}$ is the conditioning information set.

(18) $\operatorname{Var}\left(z_{t} \mid I_{t}\right)=E\left(\left[z_{t}-E\left(z_{t} \mid I_{t}\right)\right]\left[z_{t}-E\left(z_{t} \mid I_{t}\right)\right]^{\prime} \mid I_{t}\right)$

This is indeed the most appropriate definition of the uncertainty about $z_{t}$ : the conditional second moment of $z_{t}$ around the conditional mean of $z_{t} \cdot$ It is instructive, however, to first consider the unconditional variance about the conditional mean, given in (19).

$$
E\left(\left(z_{t}-E\left(z_{t} \mid I_{t}\right)\right)\left(z_{t}-E\left(z_{t} \mid I_{t}\right)\right)^{\prime}\right)=\left[I-\Sigma_{e} e_{t}^{\prime}\left(\Psi_{t} \Sigma_{e} \Psi_{t}^{\prime}\right)^{-1} \Psi\right] \Sigma_{e, t}\left[I-\Psi_{t}^{\prime}\left[\Psi_{t} \Sigma_{e} \Psi_{t}^{\prime}\right]^{-1} \Psi_{t} \Sigma_{e, t}\right]
$$

Two limiting cases arise when $\Psi_{t}$ is invertible (the full current information case) and when $\Psi_{t}$ is the zero matrix (the no current iniormation case). When $\Psi_{t}$ is invertible, the unconditional variance about the conditional mean is zero. When $\Psi_{t}$ is the zero matrix the unconditional variance about the conditional mean equals $\Sigma_{e}$, the unconditional variance about the unconditional mean. The unconditional variance about the conditional mean depends on the current information matrix $\Psi_{t}$ and on the determinants of 
the unconditional variance about the unconditional mean, $\Sigma_{e, t}$. The latter is, as was shown in equation (10), a function of the current response matrix $G_{t}^{0}$ and of the stochastic component of policy, through $\Sigma_{v}$ and $\Sigma_{u v}$. Let $\left(z_{t}-E\left(z_{t} \mid I_{t}\right)\right)\left(z_{t}-E\left(z_{t} \mid T_{t}\right)\right)^{\prime}$ be denoted by $\Lambda_{t}$. If $z_{t}$ is an n-vector, $\Lambda_{t}$ will be an $n \times n$ matrix. vec $(\Lambda)$ denotes the $n^{2} \times 1$ column vector consisting of the $n$ stacked columns of $\Lambda_{t}$, i.e. $\operatorname{vec}(\Lambda)=\left[\lambda^{1{ }^{\prime}} \lambda^{2} \cdot \cdot \cdot \lambda^{n^{\prime}}\right]^{\prime}$; $\lambda^{i}$ is the $i^{\text {th }}$ column of $\Lambda_{t}$. To obtain the conditional cxpectation of $\Lambda_{t}$ is therefore equivalent to obtaining the conditional expectation of vec $(\Lambda)$. The information set conditioning the expectation is the variance-covariance matrix of the disturbances, $\Sigma_{\epsilon}$ and the current observation $\Psi_{t} \Lambda_{t} \Psi_{t}^{\prime}$. We can express this in "vec" form by using the result that $\operatorname{vec}(A B C)=(C ' \otimes A) \operatorname{vec}(B)$ (Nissen [1968]). Thus the information set conditioning the expectation is

$$
I_{t}=\left\{\left(\left[\Psi_{t} \otimes \Psi_{t}\right] \operatorname{vec}\left(\Lambda_{t}\right)\right)^{\prime}, \operatorname{vec}\left(\Sigma_{e}\right)^{\prime}\right\}
$$

We then apply the formula for the linear least squares predictor (see Shiller [1978]):

$$
E\left[\operatorname{vec}\left(\Lambda_{t}\right)^{\prime} \mid I_{t}\right]=I_{t}\left[E\left(I_{t}^{\prime} I_{t}\right)\right]^{-1} E\left(I_{t}^{\prime}\left(\operatorname{vec}\left(\Lambda_{t}\right)\right)^{\prime}\right\}
$$

It is readily seen that the evaluation of (20) will in general be extremely messy. $E\left(I_{t}{ }^{\prime} I_{t}\right)$ and $E\left[I_{t}^{\prime}\left(\operatorname{vec}_{i}\left(\Lambda_{t}\right)\right) '\right]$ involve expectations of fourth powers of the elements of the $z$ vector. The assumption of normality of the disturbances is therefore essential for practical purposes.

The conditional variance in a model_of of optimal foreign exchange market

\section{intervention}

It is quite easy to derive the explicit solution for the two-dimensional case $\left(z_{t}=\left(z_{1 t}, z_{2 t}\right)^{\prime}\right)$ when only $z_{1 t}$ is observed concurrently. This corresponds to the case studied by Poole [1970], in which the interest rate (or money stock) is observed concurrently but real income is not. It is also 
the case studied by Boyer and by Roper and Turnovsky in which the exchange rate (or money stock) is observed concurrently but real output is not.

$$
\text { For the two variable case, } \Sigma_{e}=\left[\begin{array}{cc}
\sigma_{11} & \sigma_{12} \\
\sigma_{12} & \sigma_{22}
\end{array}\right] \text {. If the first variab]e, }
$$

$z_{1 t}$, is observed while the second one is not, $\Psi_{t}=\left[\begin{array}{ll}1 & 0\end{array}\right]$. Applying (20) we obtain the following conditional variance-covariance niatrix for $\left(z_{1 t}, z_{2 t}\right)^{\prime}$

(20') $E\left[\operatorname{vec}\left(\Lambda_{t}\right) \mid I_{t}\right]=\left[\begin{array}{l}E\left[\left(z_{1 t}-E\left(z_{1 t} \mid I_{t}\right)\right)^{2} \mid I_{t}\right] \\ E\left[\left(z_{1 t}-E\left(z_{1 t} \mid I_{t}\right)\right)\left(z_{2 t}-E\left(z_{2 t} \mid I_{t}\right)\right) \mid I_{t}\right] \\ E\left[\left(z_{1 t}-E\left(z_{1 t} \mid I_{t}\right)\right)\left(z_{2 t}-E\left(z_{2 t} \mid I_{t}\right)\right) \mid I_{t}\right] \\ E\left[\left(z_{2 t}-E\left(z_{2 t} \mid I_{t}\right)\right)^{2} \mid I_{t}\right]\end{array}\right]=\left[\begin{array}{l}0 \\ 0 \\ 0 \\ \sigma_{22}-\sigma_{12}^{2} \sigma_{11}\end{array}\right]$

The simplicity of this result is due to the fact that $z_{1 t}$ is observed concurrent1y; $E\left(z_{1 t}-E\left(z_{1 t} \mid I_{t}\right) \mid I_{t}\right)$ is therefore equal to zero. Because of this special structure, the conditional variance about the conditional mean equals the unconditional variance about the conditional mean, given by (19). Note that the conditonal variance of the unobserved variable will be strictly less than the unconditional variance, unless the errors in the reduced form equations of the observed and the unobserved variables are uncorrelated, i.e. unless $\left(\sigma_{12}=0\right) \cdot \sigma_{22}-\sigma_{12}{ }^{2} \sigma_{11}{ }^{-1}=\sigma_{22}\left[1-\rho_{12}{ }^{2}\right]$, where $\rho_{12}$ is the correlation coefficient between $e_{1}$ and $e_{2}$.

As an example, consider Boyer's model of foreign exchange market intervention. It can be summarized as follows: 


$$
\begin{aligned}
& z_{2}-a z_{1}-b G=u_{1} \\
& z_{2}+c z_{1}-d M=u_{2}
\end{aligned}
$$

$z_{2}$ is real output, $z_{1}$ the spot exchange rate, $G$ the level of government spending and $M$ the stock of money. $u_{1}$ is the goods market disturbance, $\mathrm{u}_{2}$ the money market disturbance. The exchange rate is observed concurrently, the level of output is not. There are two non-stochastic current response policy functions, relating the level of government spending and the stock of money to the exchange rate:

$$
\begin{aligned}
& G+\delta_{g^{2} 1}=0 \\
& M+\delta_{m} z_{1}=0
\end{aligned}
$$

Substituting (23) and (24) into (21) and (22) we obtain the reduced form equations

$$
\begin{aligned}
& z_{1}=\left[u_{2}-u_{1}\right]\left[a+c+d \delta_{m}-b \delta_{g}\right]^{-1} \\
& z_{2}=\left[\left(c+d \delta_{m}\right) u_{1}+\left(a-b \delta_{g}\right) u_{2}\right]\left[a+c+d \delta_{m}-b \delta_{g}\right]^{-1} .
\end{aligned}
$$

The unconditional variance of output is:

$$
E\left(z_{2}{ }^{2}\right)=\left[\frac{c+d \delta_{m}}{a+c+d \delta_{m}^{-b \delta} g}\right]^{2} \sigma_{u_{1}}^{2}+\left[\frac{a-b \delta_{g}}{a+c+d \delta_{m}^{-b \delta} g}\right]^{2} \sigma_{u_{2}}^{2}+\frac{2(c+d \delta}{\left(a+c+d \delta_{m}-b \delta_{g}\right)^{2}} \sigma_{u_{1} u_{2}}
$$

The conditional variance of output about its conditional mean, with the exchange rate, $z_{1}$, as the conditioning variable is according to $\left(20^{\prime}\right)$

$$
\begin{aligned}
E\left(z_{2}{ }^{2} \mid z_{1}\right) & =E\left(z_{2}^{2}\right)-\left[E\left(z_{1} z_{2}\right)\right]^{2}\left[E\left(z_{1}^{2}\right)\right]^{-1} \\
& =\frac{\sigma_{1}{ }^{2} \sigma_{2}{ }^{2}-\left(\sigma_{u_{1} u_{2}}\right)^{2}}{\sigma_{u_{1}}{ }^{2}+\sigma_{u_{2}}{ }^{2}-2 \sigma_{u_{1} u_{2}}}=\frac{\sigma_{u_{1}}{ }^{2} \sigma_{u_{2}}{ }^{2}\left(1-\rho_{u_{1} u_{2}}^{2}\right.}{\sigma_{u_{1}}{ }^{2}+\sigma_{u_{2}}-2 \sigma_{u_{1} u_{2}}}
\end{aligned}
$$


The conditional variance can, with a little arithmetic, be showin to be equal to the minimal value of the unconditional variance. The latter is obtained by minimizing (27) with respect to the policy instruments $\delta_{m}$ and $\delta_{\mathrm{g}}$. Thus current response policy can alter (and in general reduce) the anticipated variability of currently unobserved real income. It cannot alter the residual uncertainty (i.e. the unanticipated variance) of real income, if private agents have the same information as the public economic agent engaged in the current response policy.

The essence of this result, that the policy authorities can affect the single-period anticipated variance but not the single-period unanticipated variance should survive generalization to a greater number of instruments and state variables. Note however, that in the example under consideration, the number of unobserved variables (1) equals the number of linearly independent policy instruments (1). If the number of unobserved variables (or more generally the order of the rank deficiency of the $\Psi$ matrix) were to exceed the number of linearly independent instruments, the policy authorities will not be able to eliminate all anticipated variance. A trivial example is a "model" consisting of a single stochastic state variable and no instruments. If the state variable is observed concurrently, the singleperiod conditional variance $\operatorname{Var}\left(z_{t} \mid z_{t}\right)$ is zero. This however is not the minimized value of the unconditional variance, $\operatorname{Var}\left(z_{t}\right)$, which is independent of the non-existent instrument. If randomness is incorporated in the policy reaction function, the single-period conditional variance will depend on the stochastic component of policy. A multi-period objective functions such as $\sum_{t=1}^{T} \alpha_{t} E\left(z_{2, t}^{2} \mid I_{1}\right)$ will also yield a role for policy in reducing true uncertainty.

\section{One-_and two-period 1agged expectations_of the current statate}

To endow a macroeconomic rational expectations model, open or closed, with the inertia or sluggishness in price and quantity adjustment characteristics of many commodity and labor markets, it is necessary to introduce 
expectations of $z_{t}$ formed at several different dates bofore $t$. The procedure will be illustrated with one- and two-period forecasts of $x_{t}$. The extension to an arbitrary finite length of the forecast horizon can be found in foki and Canzoneri [1979]. The new state equation is equation ( $1^{\prime}$ ). The "policy surprise" version of the model is obtained by assuming that $x_{t}$ is again included in $z_{t} \cdot b_{1}$ and $b_{2}$ are the submatrices of $B_{1}$ and $B_{2}$ corresponding to $x_{t \mid t-1}$ and $x_{t \mid t-2}$. If $-c=b_{1}+b_{2}$, policy can only affect the bchavior of $z_{t}$ via policy forecast errors. Because of the lag in expectation formation, however, deterministic feedback control $\left(G_{t}^{1}\right)$ now can affect the single-period variance of $z_{t}$. This is because policy formation can be based on more recent information than was available to private (and public) agents when some of the expectations that influence the current state were formed.

The rational expectations solution of equations ( $\left.1^{\prime}\right)$ and (2) is most easily found by the method of undetermined coefficients. After substituting (2) in ( $1^{\prime}$ ) we obtain

$$
z_{t}=K_{0}+k_{1} z_{t-1}+K_{2} z_{t \mid t-1}+K_{3} z_{t \mid t-2}+e_{t}
$$

where

$$
\begin{aligned}
& \text { (30a) } \quad K_{0}=\left(I-C G_{t}^{0}\right)^{-1} C \bar{x}_{t} \\
& \text { (30b) } \quad \mathrm{K}_{1}=\left(\mathrm{I}-\mathrm{CG}_{\mathrm{t}}^{0}\right)^{-1}\left(\mathrm{~A}+\mathrm{CG}_{\mathrm{t}}^{1}\right) \\
& \text { (30c) } \quad \mathrm{K}_{2}=\left(\mathrm{I}-\mathrm{CG}_{\mathrm{t}}^{0}\right)^{-1} \mathrm{~B}_{1} \\
& \text { (30d) } \quad K_{3}=\left(I-C G_{t}^{0}\right)^{-1} B_{2} \\
& \text { (30e) } e_{t}=\left(I-C G_{t}^{0}\right)^{-1}\left(C v_{t}+u_{t}\right)
\end{aligned}
$$

Use the tentative solution given in (31) to derive $z_{t \mid t-1}$ and $z_{t \mid t-2}$. 


$$
z_{t}=R_{0}+R_{1} z_{t-1}+R_{2} e_{t}+R_{3} e_{t-1}
$$

Substituting these expressions for $z_{t \mid t-1}$ and $z_{t \mid t-2}$ into (29) and equating, coefficients between (29) and (31) we obtain the following expressions for $R_{0}, R_{1}, R_{2}$ and $R_{3}$ (all relevant inverses are assumed to exist).

$$
\begin{aligned}
& R_{0}=\left[I-\left(K_{2}+K_{3}\right)\right]^{-1} K_{0} \\
& R_{1}=\left[I-\left(K_{2}+K_{3}\right)\right]^{-1} K_{1}
\end{aligned}
$$

$$
R_{2}=I
$$

As pointed out by Aoki and Canzoneri [1979], the number of lagged expectations of the current price can be extended without technical complications. If the earliest forecast of $z_{t}$ in a generalization of (29) is $z_{t} \mid t-n$, the solution analogous to (31) will be a first order difference equation with an $(n-1)^{\text {th }}$ order serially correlated disturbance vector.

Equations (30), (31) and (32) make clear how the four components of a linear policy rule, current response, feedback, open-loop and stochastic, affect the probability distribution function of the $z_{t}$. The major difference between this model and the single period forecast lag model of equations (5) and (6) is that the stochastic process that characterizes the disturbances is now also affected by the feedback component of the policy rule. The matrix $R_{3}$, premultiplying the lagged disturbance term $e_{t-1}$, is a function of $G_{t}^{1}$ (as well as of $G_{t}^{0}$ ). As before we can derive the unconditional asymptotic mean and variance-covariance matrix of $z_{t}$ and the conditional and unconditional single-period means and variances. For reasons of space this is left as an exercise. 


\section{Expectations of the future}

Rational expectations models incorporating past or current expectations of the future are sjgnificantly more difficult than those incorporating only past expectations of the present--the kinds of models considered so far.

The simplest case involves forecasts no more than one period into the future. Such a model is given in equation (1"). Combining it with the policy rule (3) we obtain:

$$
z_{t}=k_{t}^{0}+k^{1} z_{t-1}+k^{2} z_{t \mid t-1}+k^{3} z_{t+1 \mid t}+e_{t}
$$

where

$$
\begin{array}{ll}
\text { (34a) } & k^{0}=\left(I-C G_{t}^{0}\right)^{-1} C \bar{x}_{t} \\
(34 b) & k^{1}=\left(I-C G_{t}^{0}\right)^{-1}\left(A+C G_{t}^{1}\right) \\
(34 c) & k^{2}=\left(I-C G_{t}^{0}\right)^{-1} B_{1} \\
\text { (34d) } & k^{3}=\left(I-C G_{t}^{0}\right)^{-1} B \\
\text { (34e) } & e_{t}=\left(I-C G_{t}^{0}\right)^{-1}\left(C v_{t}+u_{t}\right)
\end{array}
$$

Equation (33) can be used to solve for $z_{t \mid t-1}$ as a function of $z_{t+1 \mid t-1}$ :

$$
\begin{aligned}
& z_{t \mid t-1}=\left[I-K^{2}\right]^{-1} K_{t}^{0}+\left[I-K^{2}\right]^{-1} K^{1} z_{t-1}+\left[I-K^{2}\right]^{-1} K^{3} z_{t+1 \mid t-1} \\
& z_{t+1 \mid t-1} \text { in turn depends on } z_{t+2 \mid t-1}: \\
& \left.z_{t+1 \mid t-1}=\Lambda\left[I-K^{2}\right]^{-1} K_{t+1}^{0}+\Lambda\left[I-K^{2}\right]^{-1} K^{1}\left[I-K^{2}\right]^{-1} K_{t}^{0}+N\left(I-K^{2}\right]^{-1} K^{1}\right)^{2} z_{t-1}+\Lambda\left[I-K^{2}\right]^{-1} K^{3} z_{t+2} \mid t+1
\end{aligned}
$$

where $\Lambda=\left[I-\left[I-K^{2}\right]^{-1} K^{1}\left[1-K^{2}\right]^{-1} K^{3}\right]^{-1}$.

To find a unique solution for (33) therefore requires not only an initial condition $z_{0}$ for $z_{t}$, but also a condition on the expectation forma- 
tion process. With such a condition, which has frequently been chosen to be a "terminal condition" on the asymptotic behavior of the forecasting process, we can solve for $z_{t \mid t-1}$ and thus also for $z_{t+1 \mid t}$. Substituting these solutions in (33) we obtain the rational expectations solution of the model. which can now be used for policy simulation and optimization.

To save space, I shal1 discuss the non-uniqueness issue in the context of the very similar model given by equation ( ${ }^{\prime \prime \prime}$ ), which has economic agents forecasting more than one period into the future.

Combining ( $1^{\prime \prime \prime}$ ) with the policy rule (2) we obtain (35).

$$
z_{t}=k_{t}^{0}+k^{1} z_{t-1}+k^{2} z_{t+1 \mid t-1}+e_{t}
$$

where

(36a) $\quad K_{t}^{0}=\left(I-C G_{t}^{0}\right)^{-1} \overline{C x}_{t}$

(36b) $\quad \mathrm{K}^{1}=\left(\mathrm{I}-\mathrm{CG}_{\mathrm{t}}^{0}\right)^{-1}\left(\mathrm{~A}+\mathrm{CG}_{\mathrm{t}}^{1}\right)$

(36c) $\quad \mathrm{K}^{2}=\left(\mathrm{I}-\mathrm{CG}_{\mathrm{t}}^{0}\right)^{-1} \mathrm{~B}$

(36d) $\quad e_{t}=\left(I-C G_{t}^{0}\right)^{-1}\left[C_{t}+u_{t}\right]$

It is easily seen that in (35) $z_{t+1 \mid t-1}$ depends on the forecast, as of $t-1$, of $z_{t+2}$.

(37a) $z_{t+1 \mid t-1}=\left[I-K^{1} K^{2}\right]^{-1}\left[K_{t+1}^{0}+K^{1} K_{t}^{0}\right]+\left[I-K^{1} K^{2}\right]^{-1} K^{1} K^{1} z_{t-1}+\left[I-K^{1} K^{2}\right]^{-1} K^{2} z_{t+2} \mid t-1$ Similarly, $z_{t+2 \mid t-1}$ will depend on $z_{t+3 \mid t-1}$, etc.

(37b) $z_{t+2 \mid t-1}=\left[I-K^{1}\left[I-K^{1} K^{2}\right]^{-1} K^{2}\right]^{-1}\left[K_{t+2}^{0}+K^{1}\left[I-K^{1} K^{2}\right]^{-1} K_{t+1}^{0}+K^{1}\left[I-K^{1} K^{2}\right]^{-1} K^{1} K_{t}^{0}\right]$

$$
\begin{aligned}
& +\left[I-K^{1}\left[I-K^{1} K^{2}\right]^{-1} K^{2}\right]^{-1} K^{1}\left[I-K^{1} K^{2}\right]^{-1} K^{1} K^{1} z_{t-1} \\
& +\left[I-K^{1}\left[I-K^{1} K^{2}\right]^{-1} K^{2}\right]^{-1} K^{2} z_{t+3} \mid t-1
\end{aligned}
$$


Simply specifying an initial condition for $z_{t}$, say $z_{0}$, in (35) does therefore not suffice to yield a unique solution. (35) is a second-order dif-ference equation. We need an additional set of restrictions on the expectations formation process to yield a unique solution (see Taylor [1977] and Shiller [1978]). Sometimes the condition that the expectation formation process be stable suffices to find a unique solution, but this is not generally true. Another important aspect of the solution to (37) has been brought out by Shiller [1.978]. Lead (37a) by $k$ periods and take the conditiona1 expectation as of $t-1$. This gives:

$$
\begin{aligned}
z_{t \cdot 1+k \mid t-1} & =\left[I-K^{1} K^{2}\right]^{-1}\left[K_{t+k+1}^{0}+K^{1} K_{t+k}^{0}\right]+\left[I-K^{1} K^{2}\right]^{-1} K^{1} K^{2} z_{t-1+k} \mid t-1 \\
& +\left[I-K^{1} K^{2}\right]^{-1} K^{2} z_{t+2+k} \mid t-1
\end{aligned}
$$

(38) is a partial difference equation in $k$ and $t$. It therefore requires an infinite number of terminal conditions to specify the solution completely. For each $t$, we have a difference equation in $k$ which requires an approprate terminal condition for its solution. For a different $t$, this whole process needs to be repeated. In Shiller's words ". . . people in effect change their minds in each time period about terminal conditions" [Shiller, 1978, p. 26]. Clearly, the conceptual and technical problems involved here have on1y just begun to be explored. Having found a solution for $z_{t+1 \mid t-1}$ we substitute it into (35) and obtain a model which no longer contains any expectations terms.

A fairly arbitrary but simple way of cutting the Gordian knot of nonuniqueness is given by Chow [1978]. He assumes that for some sufficiently $\operatorname{large} \mathrm{T}, \mathrm{z}_{\mathrm{T}+1 \mid \mathrm{T}-1}$ is proportional or equal to $\mathrm{z}_{\mathrm{T} \mid \mathrm{T}-1}$. Substituting $\mathrm{z}_{\mathrm{T}+1 \mid \mathrm{T}-1}=$ $z_{T \mid T-1}$ into equation (35) for $t=T$, we obtain

$$
z_{T}=\left\{I+K^{2}\left[I-K^{2}\right]^{-1}\right\} K_{T}^{0}+\left\{I+K^{2}\left[I-K^{2}\right]^{-1}\right\} K^{1} z_{T-1}+e_{T}
$$

Equation (39) can now be used to derive $\mathrm{z}_{\mathrm{T}} \mid \mathrm{T}-2^{\circ}$ This yields: 


$$
\left.z_{T \mid T-2}=\left\{I+K^{2}\left[T-K^{2}\right]^{-1}\right\} k_{t}^{0}+\left\{I+K^{2}[]-K^{2}\right]^{-1}\right\} K^{1} z_{T-1} \mid T-2
$$

$\mathrm{z}_{\mathrm{T}-1} \mid \mathrm{T}-2$ is found by taking expectations of (35) for $t=\mathrm{T}-1$. We obtain

$$
z_{\mathrm{T}-1 \mid \mathrm{T}-2}=\mathrm{K}_{\mathrm{T}-1}^{0}+\mathrm{K}^{1} \mathrm{z}_{\mathrm{T}-2}+\mathrm{K}^{2} \mathrm{z}_{\mathrm{T} \mid \mathrm{T}-2}
$$

Substituting $z_{\mathrm{T}-1 \mid \mathrm{T}-2}$ from (41) into (40) we obtain $\left.\mathrm{z}_{\mathrm{T}}\right|_{\mathrm{T}-2}$ as a function of known parameters and $z_{\mathrm{T}-2}$. Ihis solution for $\left.z_{\mathrm{T}}\right|_{\mathrm{T}-2}$ is then substituted into (35) for $t=T-1$. This yields:

$$
\begin{aligned}
z_{T-1} & =\left[I+K^{2}\left[I-\left(I+K^{2}\left[I-K^{2}\right]^{-1}\right) K^{1} K^{2}\right]^{-1}\right] K_{T}^{0} \\
& +K^{2}\left[I-\left(I+K^{2}\left[I-K^{2}\right]^{-1}\right) K^{1} K^{2}\right]^{-1}\left[I+K^{2}\left[I-K^{2}\right]^{-1}\right] K^{1} K_{T-1}^{0} \\
& +\left[I+K^{2}\left[I-\left(I+K^{2}\left[I-K^{2}\right]^{-1}\right) K^{1} K^{2}\right]^{-1}\left[I+K^{2}\left[I-K^{2}\right]^{-1}\right] K^{1}\right] K^{1} z_{T-2}+e_{T-1}
\end{aligned}
$$

(42) can then be used to find $z_{T-1} \mid T-3$ (which requires the evaluation of $\left.z_{\mathrm{T}-2 \mid \mathrm{T}-3}\right)$. This solution for $\mathrm{z}_{\mathrm{T}-1 \mid \mathrm{T}-3}$ is then substituted into $\mathrm{z}_{\mathrm{T}-2}=$ $\mathrm{K}_{\mathrm{T}-2}^{0}+\mathrm{K}^{1} \mathrm{z}_{\mathrm{T}-3}+\mathrm{K}^{2} \mathrm{z}_{\mathrm{T}-1 \mid \mathrm{T}-3}+\mathrm{e}_{\mathrm{T}-2}$. In this way, equation (35) is solved backward in time. In general the equation for $z_{t}$ is

$$
z_{t}=R_{t} z_{t-1}+\sum_{i=t}^{T} D_{t, i} k_{i}^{0}+e_{t}
$$

This is also the general form of the solution for (33) if we impose the condition that for some large $T, z_{T+1 \mid T}=z_{T} \cdot R_{t}$ and $D_{t, i}$ are functions of $\mathrm{K}^{1}$ and $\mathrm{K}^{2}$ derived by repeated application of the procedure outlined in equations (37a) - (42).

Note that with $e_{t}$ given by (36d) the single-period unconditional variance of $z_{t}$ will depend on the current response component of policy, $G^{0}$ and on the stochastic components, $\Sigma_{v}$ and $\Sigma_{u v}$, but not on the feedback component $\mathrm{G}^{1}$. The asymptotic variance will however also depend on the feedback component. The single-period unconditional mean and the asymptotic mean depend 
on the current response component, the feedbark component and on the openloop component, which is embedded in $\mathrm{K}_{i}^{0}$.

\section{Conclusion}

The scope for policy in the four models given by equations (1) - (1"') can be summarized as follows. The conditional and unconditional means of the state vector $z$, whether single-period or asymptotic, wiil depend on the three non-stochastic policy components, $\mathrm{G}^{0}, \mathrm{G}^{1}$ and $\overline{\mathrm{x}}_{\mathrm{t}}$. Through its dependence on $\Sigma_{e}$, the conditional mean will also depend on the stochastic component of policy. The asymptotic variance will depend on all four policy components. The unconditional single-period variance will depend on the current response component and the stochastic component, but not on the feedback component, except in the case of model (1') which incorporates several lagged forecasts of the current value of $z$.

If variability of $z_{t}$, whether anticipated or unanticipated, is of concern to the policymaker, the unconditional variance is the appropriate focus of policy concern. If uncertainty about $z_{t}$, i.e. unanticipated variability of $z_{t}$ is what matters, the conditional variance is the appropriate object of policy destgn. In Poole's and Boyer's models the single-period conditional variance of output is independent of the current response component of policy. The design of optimal linear policy rules in stochastic dynamic rational expectations models along the lines sketched here, has applications in virtually all areas of macroeconomic stabilization policy. 
$1 /$ An important generalization of equation (2) would te to make $x_{t}$ depend on current or past predictions of the future or the present, $e \cdot g$.

$$
x_{t}=G_{t}^{0} z_{t}+G_{t}^{1} z_{t-1}+\bar{x}_{t}+v_{t}+\sum_{j=0}^{N} \sum_{i=0}^{M} G_{t}^{i j} z_{t+j} \mid t-i
$$

The analysis of the simpler equation (2) is still sufficiently complex for me not to opt for the greater generality of ( $\left.2^{\prime}\right)$ as yet. I am indebted to Pentti Kouri for bringing this up.

2/See Chow [1975] and Buiter [1979].

$3 /$ It has been objected by several colleagues that an instrument-almost by definition--is something that can be controlled without error. I would argue that it is better to model any behavioral relationship, and certainly one representing the actions of a complex, bureaucratic organization like a central bank or a treasury, as stochastic.

4/ The matrices $I-C_{t}^{0}$ and $I-\left(I-C G_{t}^{0}\right)^{-1} B_{1}$ are assumed to be of full rank.

5/ The first formal analysis of optimal foreign exchange market intervention was in Stein [1963].

$6 /$ Because of the linear dependence of the two policy reaction functions, one could be dropped without changing the analysis. The exposition stays as close as possible to Boyer's original analysis. 


\section{REFLRENCES}

I would like to thank Bill Brown for teaching me how to stack vectors. An earlier version of this paper was presented at a meeting of the NBER Research Program in International Studies in March, 1979.

[1] Aoki, M. and Canzoneri, M., "Reduced Forms of Rational Expectations Models," Quarterly Journal of Economics, 93, February 1979, pp. $59-71$.

[2] Black, Stanley W., "Comnent on J. Williamson, 'Exchange Rate Flexibjlity and Reserve Use'," Scandinavian Journal of Economics, 78, June 1976, pp. 340-345.

[3] , Floating Exchange Rates and National Economic Policy, Yale University Press, New Haven, 1977.

[4] Boyer, R.S., "Optimal Foreign Exchange Market Intervention," Journal of Political Economy, 86, December 1978, pp. 1045-1055.

[5] Buiter, Willem H., "Optimal Foreign Exchange Market Intervention with Rational Expectations," forthcoming in Trade and Payments Adjustment under Flexible Exchange Rates, J. Martin and A. Smith eds., Macmillan, 1979.

[6] Chow, G.C., Analysis and Control of Dynamic Economic Systems, John Wiley and Sons, New York, 1975.

[7] , "Econometric Policy Evaluation and Optimization under Rational Expectations," Econometric Research Program Research Memorandum No. 225, May 1978, Princeton University.

[8] Friedman, B., "Target, Instruments and Indicators of Monetary Policy," Journal of Monetary Economics, 1, November 1975, pp. 443-473. , "The Inefficiency of Short-Run Monetary Targets for Monetary Policy," Brookings Papers on Economic Activity, 2, 1977, pp. 293-335.

[10] Kareken, J.H., Muench, T. and Wallace, N., "Optimal Open Market Strategy: The Use of Information Variables," American Economic Review, 63, March 1973, pp. 156-172.

[11] LeRoy, Steve, "Efficient Use of Current Information in Short-Run Monetary Control," Special Studies Paper No. 66, Board of Governors of the Federal Reserve System, 1975.

[12] Nissen, David H., "A Note on the Varlance of a Matrix," Econometrica, 36, Jul.y-October 1968, pp. 603-604. 
[13] Poole, William, "Optimal Choice of Monetary Policy Instruments in a Simple Stochastic Macro Mode1," Quarterly Journal of Economics, 84, May 1970, pp. 197-216.

[14] Roper, D.E. and Turnovsky, S.J., "Optimal Exchange Harket Intervention in a Simple Stochastic Macro Model," unpublished, November 1978.

[15] Shiller, Robert J., "Rational Expectations and the Dynamic Structure of Macroeconomic Models: A Critical Review," Journal of Monetary Economics, 4, January 1978, pp. 1-44.

[16] Siegel, Jeremy J., "Optimal Stabilization in a General Equilibrium Financial Model," unpublished, University of Pennsylvania, October 1978.

[17] Stein, J.L., "The Optimum Foreign Exchange Market," American Economic Review, 53, June 1963, pp. 384-402.

[18] Taylor, John, "On Conditions for Unique Solutions in Stochastic Macroeconomic Models with Price Expectations," Econometrica, 45, November 1977, pp. 1377-1385.

[19] Turnovsky, Stephen J., "The Relative Stability of Alternative Exchange Systems in the Presence of Random Disturbances," Journal of Money, Credit and Banking, 8, February 1976, pp. 29-50.

[20] Woglom, G., "Rational Expectations and Monetary Policy in a Simple Macroeconomic Model," Quarterly Journal of Economics, 93, February 1979, pp. 91-105. 